\title{
Celda para Medición de la Evaporación Real en Suelo Desnudo no Saturado
}

\author{
Péricles Alves Medeiros \\ Departamento de Ingeniería Sanitária y Ambiental / CTC / Universidade Federal de Santa Catarina, Florianópolis / SC - BRASIL. \\ pericles@ens.ufsc.br
}

Recebido: 14/07/03 - revisado: 19/12/03 aceito: 20/05/05

\section{RESUMEN}

Este trabajo trata de evaporación real de suelo desnudo no saturado y complementa una investigación anterior sobre balance hidrico en relleno sanitario con basura domiciliaria urbana, en escala casi real. Una celda portable fue diseñada y desarrollada especialmente para determinar aquel primer parámetro a través de pesajes sucesivos con balanza de precisión. Asimismo, un pequeño evaporímetro de tanque fue construido para determinar la evaporación del água. Los resultados de los dos aparatos fueron constrastados con la serie histórica del tanque "Clase A" de la estación meteorológica más cercana. La evaporación de suelo desnudo también fue determinada a través de tres sencillas simulaciones matemáticas. En ese caso, se utilizaron adecuados coeficientes de tanque y de cultura para el estadio inicial justo después de una sembradura. Una búsqueda en la literatura posibilitó una amplia comparación de resultados medidos en distintos climas. Por fin, se considera que la celda a pesar de su bajo coste, presentó funcionamiento totalmente satisfatório.

Palabras-clave: evaporación de suelo desnudo; modelos físicos; lisímetros.

\section{INTRODUCCIÓN}

Dentro del ciclo hidrológico, uno de los procesos más complejos y de dificil monitoreo exato es la evapotranspiración. Hay muchos tipos de suelos, manejos, vegetación y climas. Tucci (1993) afirma que: "informaciones confiables sobre evapotranspiración real son escasas y de difícil obtención ya que demandan un largo tiempo de observação y costan muy caro". Para proyectos de regadios ese parámetro es importante pues, en muchos casos, es muy util en la determinación de la necesidad hídrica de un cultivo. La evapotranspiración depende del clima y además, de las propiedades del perfil del suelo. En ese último caso, se tiene realmente algunos parámetros de difícil determinación. Una manera de medir el poder evaporativo de la atmosfera, es la utilización evaporímetros como los atmómetros y tanques de evaporación. En los primeros, hay un recipiente con água conectado a una superfície porosa como papel, porcelana, etc. Eses, son baratos, pequenos y portables, pero de poca confiabilidade. Como ejempos, se tiene el evaporímetro de Piché, Livinstone y Bellani. En los tanques, el água evapora livremente a través de su superfície. Existen muchos modelos de tanques pero aqui se utilizarán dos tipos: el modelo "Clase A" del United States Weather Bureau por ser de gran utilización en el mundo y un pequeño de 9,0 litros. El tanque Clase A tiene 1,21 metros de diámetro interno y, aparte de la variación de nivel del água, hay que medirse también la lluvia ocurri- da. Para su adecuada operación necesita mantenimiento, reposición de água y cuidados con los animales que pueden beber el água. A veces, debido a un aguacero, corren el riesgo de rebozamiento invalidando la lectura del nivel del água. Para la determinación de la evaporación de un lago o la evapotranspiración de referencia, se corrige los resultados del dicho tanque con coeficientes empíricos adecuados a cada caso. Vários autores estableceron modelos matemáticos de variado grado de complejidad, o fórmulas para determinación de la evapotranspiración en distintas situaciones. La precisión de los resultados, muchas veces no son buenas incluso en modelos muy elaborados exactamente por exigiren un gran numero de datos a veces de cara obtención. Registra-se aqui solamente algunos colaboradores más clásicos como Penman (1948), Monteith (1965), Jensen (1973), Doorembos et al.(1977), Ritchie ( 1972), Blaney-Criddle (1950), Hargreaves (1994), Thornthwaite (1939), etc. Más adelante, en la Tabla 1, para comparación de resultados, se presenta otros autores más actuales como por ejemplo Yuen et al (2001). En ese estudio, el autor presenta resultados de la aplicación del modelo HELP - Schroeder et al (1994) y modelo de Jensen et al (1970, 1971, 1990).

En cuencas reales, se puede separar la evapotranspiración como incógnita dentro de un balance hídrico global. En ese caso, la lluvia y el escurrimiento superficial son monitorados en largo tiempo.

Con el control de la humedad en várias camadas del suelo también se puede obtener la evapotranspiración 
por sucesivos mostreos de ese parámetro. Para la medición de la humedad se puede utilizar el tensiómetro, balances hídricos con pesajes de muestras del suelo, sonda de neutrones, TDR, etc.

La evapotranspiración real de un suelo (ETR) puede ser obtenida indirectamente a través de un balance hídrico en un lisímetro. Este, resumidamente, es un volumen cerrado de suelo no alterado y en su sitio mismo. El monitoreo periodico del grado de humedad, lluvia ocurrida o riego artificial, líquidos percolados y, a veces, peso total del aparato (o presión sobre neumáticos ), permite la determinación de la ETR como incógnita. En este trabajo, para comparaciones, se utilizaron mediciones hechas por otros autores en distintos tipos de lisímetros, microlisímetros o celdas diversas. A continuación, un resumen de algunos trabajos utilizados con esa técnica. Mellouli et al (2000) investigaron las pérdidas por evapotranspiración en suelo desnudo en un cultivo de almendras en en sul de España y outro, de olivos, en Tunísia. Los autores utilizaron celdas de 48,5 por 48,5 por $50,0 \mathrm{~cm}$. En ese caso, la lluvia fue artificial con simulador. La evaporación real del suelo desnudo fue determinada por una integración numérica de varias camadas en las cuales la humedad era medida con la técnica de sondas de TDR (Time Domain Reflectometry). Ese proceso considera una relación entre la constante dielétrica aparente del suelo y la humedad volumétrica. Menziani et al (1999) en el Lido de Venezia, estudiaron la evaporación de la fina arena de la playa con un lisímetro enterrado de 3141,6 litros de volumen. La investigación empezó con el suelo totalmente saturado y la evaporación es determinada por una integración de la várias camadas de arena. En ese caso, también se utilizó la TDR.. Jackson et al ( 1999) en Kenia, usó 24 micro-lisímetros de 2,0 litros en suelo desnudo bajo sombra de árboles y, también, bajo sol. Los pequenos lisímetros fueron pesados diariamente para el cálculo de su balance hídrico. Mwendera et al (1997), en Bélgica estudió la influencia de dos tipos de manejos de la superfície de suelo desnudo en la evaporación. Aqui se utilizaron muestras con micro-llisímetros de 5,0 litros, pesadas en laboratório. Se calculó la evaporación por integración numérica de 7 camadas de suelo.Plauborg (1995) en Dinamarca, con dos métodos: TDR y muestras de micro-lisímetros de 0,8 litros, estudió la evaporación de suelo desnudo en campo experimental. Las pequeñas celdas eran usadas para la retirada sistematica de muestras, pesajes y posterior retorno a su sítio original.Wallace et all (1999), en Kenia, trabajaron con sucesivos pesajes de micro-lisímetros de 0,8 litros determinando la misma comentada evaporación con y sin sol. Los autores utilizaron las mediciones para elaborar un modelo matemático. Qiu et al (1998), en Japón, también con micro-lisímetros de 18,0 y 78,0 litros, hicieron un modelo matemático relacionando evaporación con temperatura de la superfí́cie del suelo. Pauliokonis et al (2001),en USA, estudiaron evapo- ración de suelos saturados de un lago con y sin vegetación aquatica. Fueron utilizados lisimetros de 34,3 litros de volumen. Yuen et al (2001) en Australia, investigaron el balance hidraulico de un relleno sanitário real. En este caso, utilizaron modelos matemáticos de Jensen (1970, 1971, 1990) y el modelo H.E.L.P. evaluado por Schroeder et al (1994). Para el balance, la evapotranspiración de la camada de suelo de cobertura fue determinada por los referidos modelos. Los autores contrastaron los resultados calculados con la evapotranspiación potencial del sitio. Daamen et al (1995) en región semi árida de Níger, utilizaron muestras de suelo con el método de micro-lisímetros de 1,8 y 0,2 litros para la determinación de la evaporación de suelos. Otro caso puede ser el suelo de cobertura cerrando un relleno sanitário. Este último, fue el caso del presente trabajo en lo que se medió con un modelo reduzido (celda) la evaporación real de un relleno sanitário de laboratório cubierto con arena gruesa sin vegetación. Más adelante, se continua y desarrolla especificamente este tema.

Como evapotranspiración potencial se entiende la máxima producida por un suelo con vegetación baja cubriendo todo el area y, siempre con suficiente humedad. La definición exacta de evapotranspiración potencial no es tan sencilla y varia segun el autor y metodologia adoptada. Asi que muchos autores reemplazan ese parámetro por otro más adecuado que es la "evapotranspiración de referencia” (ETo) a seguir comentada. Esa puede ser relativa a alfafa o césped, bajo determinadas condiciones. Aparte de los sofisticados modelos matemáticos comentados, el más sencillo es lo del tanque "Clase A". Con sus datos, se puede llegar a la evapotranspiración de referencia (ETo) y de una determinada cultura a través de las fórmulas:

$$
\mathrm{ETo}=\mathrm{Kt} . \mathrm{ECA}
$$

$$
\mathrm{ETPc}=\mathrm{Kc} . \text { Eto }
$$

Todos los simbolos están agrupados en seción específica más adelante. Según Dororenbos and Pruitt (1977), el valor de Kt , sitúase en el rango de 0,35 hasta un 0,85 . En la gran mayoria de los casos, varia de 0,50 hasta 0,80. Jensen (1974), Dororenbos y Pruitt (1977), y Hounam (1973) discuten otros fatores adicionales que influencian la evaporación del tanque "Clase A" como por ejemplo, el color de la pintura. El ciclo de crescimiento de una planta produce distinas demandas de água. Normalmente se divide ese desarrollo en 4 estadios: $1^{\circ}$ ) Inicial, $2^{\circ}$ ) Vegetativo, $3^{\circ}$ ) Floración y $4^{\circ}$ ) Maturación. En el primer período la evapotranspiración es praticamente igual a la evaporación de suelo desnudo. En ese caso, se produce la 
evapotranspiración mínima. En los primeros dias, la evapotranspiración sitúase, en general, entre $25 \%$ y $65 \%$ de la ETo. Con el desarrollo del cultivo, llega a 100 $\%$ (muchas veces incluso un poco más). La Figura 1 enseña la variación típica del coeficiente de cultura $(\mathrm{Kc})$.

\section{ORIGEN DE ESTE TRABAJO}

Este trabajo es praticamente un sub producto de una otra amplia investigación de laboratório ubicada en el campus de la UFSC, en Florianópolis-SC. Fue desarrollado en del marco del Programa de Saneamento Básico, PROSAB III desarrollado en 2001: "Tecnologias de Apoio ao Desenvolvimento de Aterros de Resíduos Urbanos para Pequenos Municípios”. Ver Medeiros et al (2002-a), Medeiros et al (2002-b), Medeiros et al (2002-c). Para una referencia a ese proyecto mayor, en ese texto, se utilizará la expresión: "proyecto de orígen". Ese fue un aparato experimental con 3 celdas de 1,54 × 1,57 x 3,35 metros cada, y rellenadas con basura domiciliaria urbana de Florianópolis. Arriba de la basura, se colocó una capa de arena gruesa con 300 $\mathrm{mm}$ de espessor. Entre los vários objetivos de dicho proyecto, lo que interesa aqui es el balance hidráulico de las dichas celdas. Más concretamente, dentro de ese balance el mayor problema fue como medir la_evaporación real del conjunto. La importancia de la determinación de la evaporación (o evapotranspiración si hubiera alguna vegetación) de un relleno sanitario está basada en, por lo menos, dos aspectos. Primero, porque ese parámetro es absolutamente necesário para la realización del balance hídrico del sistema que está integrado con el ambiente abierto. Por último, según Maurer (1994), Krol et al (1994), la moderna tendencia de proyecto de un relleno es considerarlo como una "wet cell" es decir, un biorreactor. A veces, los líquidos percolados son recirculados para acelerar las decomposición. Continua el autor: "...un mejor conocimiento de la hidrologia de un proyecto de relleno sanitario por lo tanto, revelase más crucial ya que la decomposición de la basura, tratamiento local del lixiviado y producción de gas están muy relacionados con el grado de humedad".

\section{DISEÑO Y OPERACIÓN DE LA CELDA}

De este punto para adelante, se utilizará la palavra "celda" para definir el modelo reduzido destinado a medir la evaporación real del "proyecto de orígen". La determinación de la evaporación real de un suelo desnudo, no saturado, con los modelos matemáticos existenes o fórmulas sencillas, no es una tarea fácil. Depende de tipo de suelo, espesor, grado de adensamiento, perfil de humedad disponible, clima, vientos, temperatura, radiación, cercanias del experimento, etc. Por todo ello, el resultado muchas veces no es muy seguro. Para su validación, siempre se necesitaria de contraste con datos experimentales realmen- te observados. Esta investigación está direccionada a esa segunda via. Es decir, se optó por un modelo físico en escala reducida en el cual la evaporación fuera determinada por pesajes sucesivos. Asi que la idea fue de un tipo de lisímetro, o sea, de un volumen cerrado y totalmente controlado. Aqui se pone de manifiesto que este diseño es original y no fue adaptado de ningún otro. Aunque pueda ser mejorado o criticado, es único.

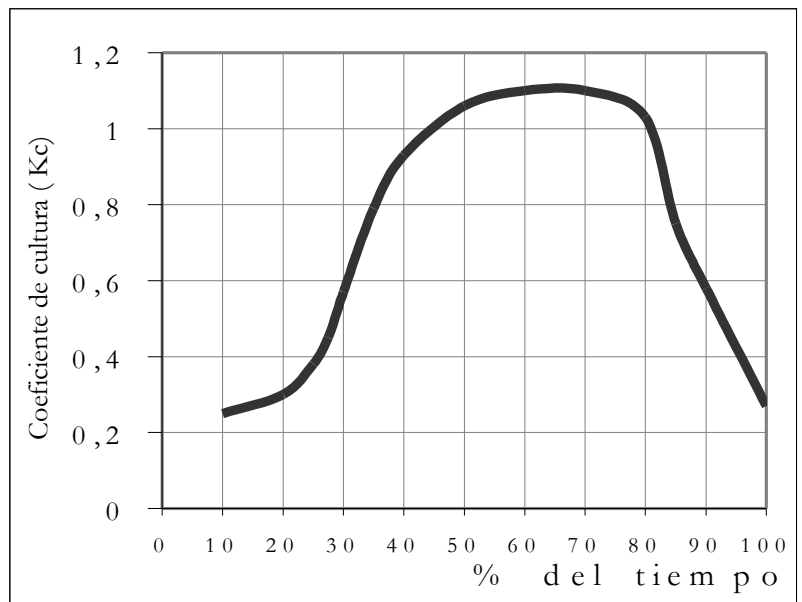

Figura 1 - Evolución típica del coeficiente de cultura de la sembradura a la cosecha.

El primer problema fue el tamaño adecuado. Lo mejor modelo seria, al principio, el más grande y, por ello, el más representativo de la realidad. Desafortunadamente, se verificó que la precisión de las balanzas disminuyen con la carga. En este caso, por tratarse de una variable muy sensível, necesitamos de una gran precisión y, por ello, la carga no podria ser muy grande.Considerando el corto presupuesto y los equipos disponibles, se utilizó finalmente una balanza piezoelétrica digital con capacidad máxima de $50 \mathrm{~kg}$ y precisión de 2,0 gramos (error máx de 0,01\%). El paso siguiente fue la forma de la celda. El plato de la balanza solo tenia 35 por $45 \mathrm{~cm}$. Asi que la base del aparato tendria que ser pequeña. Como la balanza necesita proteción tubo que quedar un poco lejos y asi, para cada pesaje habria que realizr un pequeño transporte. Asi que, aparte, del peso, la celda tendria quer ser facilmente transportable Por todo ello, al final, se elegió el cilindro como mejor forma. Un tuberia de PVC de 200,3 mm de diámetro externo fue el escogido. Dentro, fue colocado una camada de la dicha basura de $300 \mathrm{~mm}$ de espesor y, arriba, una de capa de arena gruesa también con $300 \mathrm{~mm}$ de altura. Es decir, la cobertura quedó con espesor exatamente igual al "proyecto de orígen". La arena posee $\mathrm{d}_{50}=0,75 \mathrm{~mm}$, coeficiente de uniformidad $\left(\mathrm{d}_{60} / \mathrm{d}_{10}\right)=4,27$ y masa específica aparente húmeda de $1350 \mathrm{~kg} \mathrm{~m}^{-3}$. 


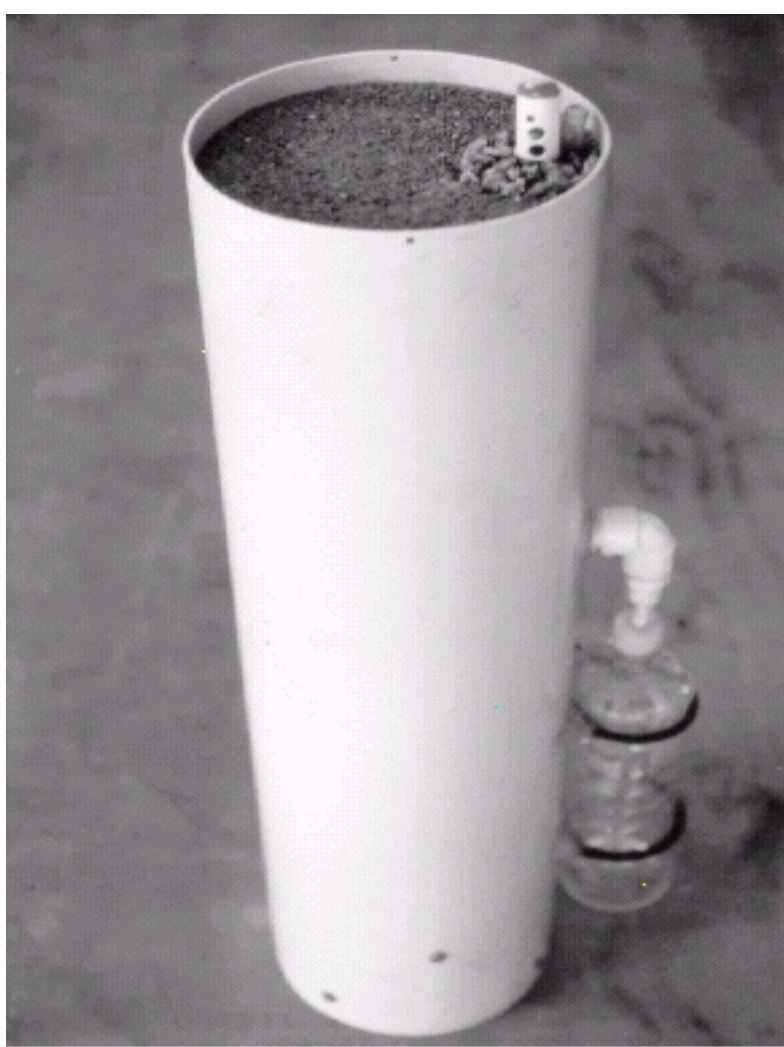

Figura 2 - Montaje final de la celda

Debido al tranporte y manejo, el peso de la celda tube que ser lo menor posible dentro de la representatividad deseable. Los principales datos de la composición gravimétrica de la basura, en el dia "zero" (07/06/2001), eran: $57,56 \%$ de material orgánico; $6,85 \%$ de papel; 3,09 $\%$ de cartón; $9,94 \%$ de plástico blando; $2,42 \%$ de plástico duro; $4,68 \%$ de trapos y cueros; $8,69 \%$ de pañales y basura sanitaria y $6,77 \%$ de otros materiales. Los grados de humedad inicial de la basura y de la arena (en peso) fueron de 55,2 y $4,75 \%$ respectivamente. Las masas específicas aparentes húmedas fueron: 1350,00 y 1322,48 $\mathrm{kg} \mathrm{m}^{-3}$ respectivamente para arena y basura. Abajo de la basura, para sangrias, se diseñó un pequeño embalse y bajo presión atmosférica. La altura total de la celda resultó siendo 700 $\mathrm{mm}$. Para la escorrentía superficial, fue diseñado un sistema con ajuste de la entrada de água superficial debido a los asentamientos de la materia orgánica y basura. Aqui se presentan tan solamente las soluciones finales y no la mayor parte del trabajo que fue el desarrollo de la idea inicial. Por fin, un otro sistema mantiene la presión del embalse inferior siempre igual a la atmosferica pero sin permitir la entrada de lluvia para el embalse. En este balance no se consideró el arrastre de gases junto con el vapor de água. Asimismo, del líquido sangrado del embalse no se retiró la matéria sólida disuelta. Es decir, el balance considera que toda la materia evaporada o drenada sea $100 \%$ líquido. La
Figura 2 enseña una fotografía final de la celda. Con relación a precipitación, al principio, se pensó en lluvia artificial. Se tendria que elegir un hietograma de proyecto o algun tipo de investigación sobre lluvias intensas de la región. Por fin, se elegió la lluvia natural y la celda fue colocada sobre el suelo, justo al lado del "proyecto de orígen" sometiéndose a la intempérie. El suelo de la cercanía era desnudo y la distancia mínima hasta el borde fue cerca de 10 metros. Asi que las condiciones del experimento son de una capa de arena sobre un relleno sanitário. El tanque Clase A más cercano es rodeado de césped y ubicado a $15 \mathrm{~km}$ de distancia como se explicó anteriormente. En este estudio preliminar no se desarolló ninguna corrección adicional respecto a detalles de las condiciones de borde, que no sean las originales de Doorenbos y Pruitt (1977) explicadas más adelante.

\section{METODOLOGIA}

El resumen de la metodologia elegida es:

1) En el "tiempo zero" se tiene un volumen cerrado con condiciones iniciales conocidas: grado de humedad de la arena y de la basura igual al "proyecto de orígen" y peso total del aparato completo (18295 gramos).

2) En el "tiempo 1" (en general, después de 4 a 7 dias) la celda es pesada otra vez .

3) La lluvia ocurrida en ese período es medida en un pluviómetro al lado del experimento.

4) Una ecuacíon de balance hidrico determina la evaporación real del período como incógnita.

5) La evaporación medida del período es dividida por el numero de dias.

6) Repite-se el procedimento pero siempre considerando las alteraciones de peso producidas por sangrias del embalse inferior o pequeños arreglos.

\section{Lista de Símbolos}

ECA evaporación de tanque "clase A", $\left(\mathrm{mm} \mathrm{dia}^{-1}\right)$.

ETo evapotranspiración de referencia, $\left(\mathrm{mm} \mathrm{dia}^{-1}\right)$.

ETPc evapotranspiración potencial de la cultura, $\left(\mathrm{mm} \mathrm{dia}^{-1}\right)$

ETR evapotranspiración real de la cultura, si hubiera baja disponibilidad hídrica: ETR es menor o igual a ETPc. (mm dia-1) evaporación de suelo desnudo, simulada a diario

K t coeficiente de tanque "clase A"

K c coeficiente de la cultura

$\mathbf{d}_{1}$ diámetro interno del cilindro: $194,9 \mathrm{~mm}$.

$\mathbf{d}_{2}$ diámetro medio $\left[\left(\mathrm{d}_{1}+\mathrm{d}_{3}\right) / 2=197,6 \mathrm{~mm}\right.$ ]

$\mathbf{d}_{3}$ diámetro externo del cilindro $(=200,3 \mathrm{~mm})$

$\mathbf{A}_{1}$ area $\pi\left(\mathrm{d}_{1}\right)^{2} / 4\left(=29834,14 \mathrm{~mm}^{2}\right)$

$\mathbf{A}_{2}$ area $\pi\left(\mathrm{d}_{2}\right)^{2} / 4\left(=30666,47 \mathrm{~mm}^{2}\right)$ 
$\mathbf{A}_{3}$ area $\pi\left(\mathrm{d}_{3}\right)^{2} / 4\left(=31510,24 \mathrm{~mm}^{2}\right)$

$\mathbf{P}$ precipitación acumulada del período, en $\mathrm{mm}$.

E evaporación acumulada del período, en $\mathrm{mm}$.

E* evaporación de suelo desnudo diária média del período, en mm.dia ${ }^{-1}$

n número de dias de 1 período (aprox. 4, 5, 6,7 dias)

$\mathbf{N}$ número de ordem

$\mathbf{W}_{\mathbf{i}}$ peso total de la celda, en el tiempo “i”, en gramos.

$\mathbf{W}_{\mathrm{i}-1}$ lo mismo, en el tiempo anterior, en gramas.

F peso acrecido (+) o retirado ( - ) del modelo, en el período, debido sangria del embalse y arreglos (g)

ESC escorrentía superficial acumul. del período, en $\mathrm{mm}$

I infiltración acumulada del período, em $\mathrm{mm}$

a, b coef. lineal y angular de la reta ajustada de B. Criddle

p porcentaje de horas con sol (B. Criddle)

t temperatura media mensual $\left({ }^{\circ} \mathrm{C}\right)$

Ic Indice de calor (Thornthwaite)

fc fator de correção en función de latitud y mes

\section{Determinación de la evaporación media diária}

Por balance hídrico en la celda, la fórmula final para el cálculo de la evaporación media diaria en cada período, en $\mathrm{mm} \mathrm{dia}^{-1}$ es:

$$
\mathrm{E}^{*}=\left[0,03352\left(30,66647 \mathrm{P}+\mathrm{W}_{\mathrm{i}-1}-\mathrm{W}_{\mathrm{i}}+\mathrm{F}\right)\right] / \mathrm{n}
$$

\section{RESULTADOS Y DISCUSIÓN}

En realidade, la celda fue monitoreada por 2 años en el "proyecto de orígen". En este artículo, se enseñaron los resultados del 07/Jun/2001 al 06/Nov/2001. Más adelante, para contrastes, se utilizó tan solo del 08/Jun/2001 hasta el 30/Sep/2001, ambos inclusive (115 dias consecutivos). Eso es para la coicidencia de tiempo entre datos de la celda y 2 tipos de evaporímetros, disponibles en aquel entonces. La Tabla 2 enseña en detalles todo el monitoreo. Para el cálculo de la última coluna se utilizó la ecuación (3). Segun Nerilo et al (2002), la máxima intensidad de lluvia en la región fue $424,1 \mathrm{~mm} \mathrm{~h}^{-1}$, con tiempo de retorno de 100 años y duración 5 minutos, en la vecina Paulo Lopes-SC. Por otro lado, un ensayo de infiltración hecho en el Laboratório de Hidráulica del Departamento de Ingeniería Sanitária Y Ambiental de la UFSC, reveló que la arena utilizada para cierre de la celda posee una capacidad máxima de infiltración de $680,0 \mathrm{~mm} \mathrm{~h}^{-1}$. Es decir, la probabilidad de ocurrir escurrimiento superficial era pequeña. De hecho, no ocurrió (ver Tabla 2). De todas formas, la celda, a principio, fue concebida para manejar escorrentía y ser aplicada a cualquier suelo. Las evaporaciones máxima y mínima resultaron en 3,016 y $0,548 \mathrm{~mm} \mathrm{dia}^{-1}$, respectivamente. La media general fue de $0,967 \mathrm{~mm} \mathrm{dia}^{-1}$. Se percibe que la evaporación media de un cierto "período"(entre 2 mediciones) es directamente proporcional a la lluvia ocurrida, pero no es el único fator influyente. A veces, el aumento de la evaporación trás algunas lluvias, no se produce imediatamente sino que después de 3 o 4 dias de retardo. Para una explicación más completa, habria que colectar datos de vientos, temperatura, nubosidad, radiación solar y humedad relativa del aire. Hay que dejar muy claro que un monitoreo completo de datos climáticos no fue el objetivo principal del presente estudio. La Figura 3 enseña las evaporaciones medidas en los 115 dias de observación para: Celda (el modelo reducido), tanque Clase A y "tanque 1". Como los datos del tanque Clase A fornecidos por el INMET presentabon algunas fallas, se hizo un relleno con las medias mensuales, mes a mes. Por todo ello, se percibe que algunos pontos tienen exactamente la misma ordenada. El evaporímetro llamado "tanque 1" fue instalado para confirmaciones y contraste entre distintos resultados. Era un cilindro de PVC con $214 \mathrm{~mm}$ de diámetro interno y $250 \mathrm{~mm}$ de altura dotado de un sistema de medición de niveles. Para mejorar la precisión, se leía el nivel del água siempre con una lente de $100 \mathrm{~mm}$ de diámetro lo que permitia percibir algo como $1 / 4$ de $\mathrm{mm}$. El tanque Clase A más cercano adoptado pertenece a la estación meteorológica "Florianópolis no 83897 ", código 02748006 DNAEE (1996), ubicada en São José-SC, a 15 $\mathrm{km}$ de distancia del experimento. Actualmente esta bajo responsabilidad del $8^{\circ}$ Distrito de Meteorologia del INMET. Los datos principales de evaporación están enseñados en la Tabla 1 que incluye las simulaciones matemáticas explicadas más adelante. Considerándose el tanque Clase A como base (el $100 \%$ ), el "tanque 1" representaria, en promedio, un $62,7 \%$ de la evaporación de superfície líquida libre y, la celda, un 23,6\%.. El tanque Clase A presentó evaporación máxima maximorum de 7,63 $\mathrm{mm}$ y, la mínima minimorum de $1,48 \mathrm{~mm}$. Respectivamente, el tanque 1 y celda presentaron: 6,500-0,430 y 2,180-0,548 $\mathrm{mm}$. La relación de áreas entre el los dos evaporímetros es 32. Hay que señalar que el tanque Clase A es de latón y el "tanque 1" es de PVC. Un estudio de la influencia del material del tanque, area, etc, está fuera del actual objetivo . Adelante, la Figura 4 enseña, acumuladamente, los datos de la Figura 3 juntamente con la lluvia ocurrida y 3 sencillos modelos matemáticos a continuación explicados.

Tabla 1 - Datos principales de evaporación

\begin{tabular}{llll}
\hline \hline Equipo/Autor & $\begin{array}{l}\text { Promedio } \\
\left(\mathrm{mm} \mathrm{dia}^{-1}\right)\end{array}$ & $\begin{array}{l}\text { Total } \\
(\mathrm{mm})\end{array}$ & $\begin{array}{l}\% \\
\text { (relativ) }\end{array}$ \\
\hline Celda & 0,97 & 111,3 & 23,6 \\
Tanque1 & 2,57 & 295,7 & 62,7 \\
Tanque Classe A & 4,10 & 471,1 & 100,0 \\
Simul. C/Classe A & 0,93 & 107,4 & 22,8 \\
Simul. C/B.Criddle & 2,98 & 342,5 & 72,7 \\
Simul. C/Thorntwaite & 1,51 & 173,9 & 36,9 \\
\hline \hline
\end{tabular}


Tabla 2 - Monitoreo de la celda

\begin{tabular}{|c|c|c|c|c|c|c|c|c|c|}
\hline $\mathbf{N}$ & $\begin{array}{l}\text { Tiempo } \\
\text { (dias) }\end{array}$ & Fecha y 1 & & $\begin{array}{l}W_{i} \\
(g)\end{array}$ & $(\mathrm{mm})$ & $\begin{array}{l}\text { ESC } \\
(\mathrm{mm})\end{array}$ & $\begin{array}{c}\text { I } \\
(\mathrm{mm})\end{array}$ & $\begin{array}{l}E \\
(\mathrm{~mm})\end{array}$ & $\begin{array}{l}\text { E* }^{*} \\
(\mathrm{~mm})\end{array}$ \\
\hline 0 & ....... & 07/Jun & $14: 40$ & 18295 & & & & & \\
\hline 1 & 06 & 13/Jun & $15: 00$ & 18196 & 1,124 & 0,00 & 1,124 & 4,474 & 0,746 \\
\hline 2 & 05 & 18/Jun & $14: 45$ & 18542 & 14,183 & 0,00 & 14,183 & 2,981 & 0,596 \\
\hline 3 & 04 & 22/Jun & $15: 00$ & 18909 & 16,032 & 0,00 & 16,032 & 4,178 & 1,044 \\
\hline $3^{\mathrm{A}}$ & $\ldots \ldots$ & $27 /$ jun & $16: 00$ & ( - ) 1974 & & .............. & & ................ & ……...... \\
\hline 4 & 07 & 29/Jun & $15: 00$ & 19280 & 85,671 & 0,00 & 85,671 & 9,460 & 1,351 \\
\hline 5 & 05 & 04/Jul & $15: 40$ & 19233 & 1,440 & 0,00 & 1,440 & 3,055 & 0,611 \\
\hline 6 & 06 & $10 / \mathrm{Jul}$ & $16: 20$ & 19267 & 5,414 & 0,00 & 5,414 & 4,425 & 0,737 \\
\hline $6^{\mathrm{A}}$ & $\ldots \ldots$ & $23 / \mathrm{Jul}$ & 09:15 & ( - ) 984 & & & & & \\
\hline 7 & 13 & 23/Jul & $14: 45$ & 19243 & 56,240 & 0,00 & 56,240 & 25,631 & 1,972 \\
\hline 8 & 14 & 06/Ago & $14: 30$ & 19842 & 27,969 & 0,00 & 27,969 & 8,762 & 0,619 \\
\hline 9 & 04 & 10/Ago & $15: 20$ & 19756 & 0,000 & 0,00 & 0,000 & 2,883 & 0,721 \\
\hline 10 & 06 & 16/Ago & $15: 00$ & 19654 & 0,245 & 0,00 & 0,245 & 3,671 & 0,612 \\
\hline 11 & 07 & 23/Ago & $16: 00$ & 19806 & 9,569 & 0,00 & 9,569 & 4,741 & 0,677 \\
\hline $11^{\mathrm{A}}$ & $\ldots \ldots$ & 30/Ago & $14: 00$ & ( - ) 989 & ................. & ........... & & & ................ \\
\hline 12 & 07 & 30/Ago & $14: 30$ & 18942 & 9,077 & 0,00 & 9,077 & 5,140 & 0,734 \\
\hline 13 & 04 & $03 /$ Set & $14: 17$ & 19077 & 8,476 & 0,00 & 8,476 & 4,187 & 1,047 \\
\hline $13^{\mathrm{A}}$ & ........ & 03/Set & $15: 30$ & $(+) 73$ & ……............ & & ……....... & ............... & ……...... \\
\hline 14 & 03 & 06/Set & $15: 30$ & 19827 & 28,440 & 0,00 & 28,440 & 6,541 & 2,180 \\
\hline $14^{\mathrm{A}}$ & $\ldots \ldots$ & 06/Set & $16: 20$ & $(+) 73$ & ...................... & 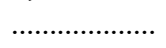 & ....................... & 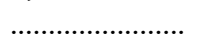 & ............... \\
\hline 14B & $\ldots \ldots$. & 13/Set & $09: 50$ & ( - ) 1366 & .................. & 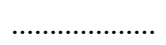 & 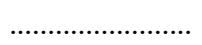 & 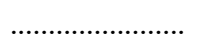 & .................. \\
\hline 15 & 07 & 13/Set & $10: 20$ & 19324 & 32,715 & 0,00 & 32,715 & 7,148 & 1,021 \\
\hline 16 & 04 & $17 /$ Set & $15: 30$ & 19512 & 9,983 & 0,00 & 9,983 & 3,960 & 0,990 \\
\hline 17 & 03 & $20 /$ Set & $15: 30$ & 19480 & 1,281 & 0,00 & 1,281 & 2,389 & 0,796 \\
\hline 18 & 04 & 24/Set & $14: 30$ & 20482 & 37,011 & 0,00 & 37,011 & 4,458 & 1,114 \\
\hline $18^{\mathrm{A}}$ & ....... & 01/Out & $14: 00$ & ( - ) 3858 & …................. & ........... & ……........ & ............. & ............. \\
\hline 19 & 07 & 01/Out & $15: 30$ & 20488 & 129,735 & 0,00 & 129,735 & 3,838 & 0,548 \\
\hline 20 & 03 & 04/Out & $16: 40$ & 20373 & 12,770 & 0,00 & 12,770 & 9,049 & 3,016 \\
\hline $20^{\mathrm{A}}$ & $\ldots \ldots$ & 04/Out & $16: 50$ & ( - ) 134 & ..................... & ............ & 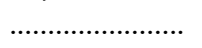 & ............... & $\ldots .$. \\
\hline $20 B$ & $\ldots \ldots$. & 09/Out & $15: 00$ & ( - ) 2240 & & & & & \\
\hline 21 & 05 & 09/Out & $15: 30$ & 19496 & 57,597 & 0,00 & 57,597 & 9,026 & 1,805 \\
\hline $21^{\mathrm{A}}$ & ........ & 09/Out & $16: 50$ & $(+) 38$ & ……........... & 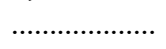 & ……................ & …….............. & . \\
\hline $21 B$ & ...... & 18/Out & $15: 00$ & $(-) 444$ & …................ & & & & \\
\hline 22 & 09 & 18/Out & $17: 00$ & 19432 & 21,603 & 0,00 & 21,603 & 10,742 & 1,193 \\
\hline $22^{\mathrm{A}}$ & ...... & 18/Out & $17: 45$ & $(+) 3$ & & & & & \\
\hline 23 & 06 & 24/Out & $16: 00$ & 20390 & 40,044 & 0.00 & 40,044 & 9,151 & 1,525 \\
\hline $23^{\mathrm{A}}$ & ........ & 24/Out & $16: 15$ & ( - ) 1335 & ...................... & $\ldots \ldots$. & ………....... & ................ & ............. \\
\hline 24 & 05 & 29/Out & $14: 30$ & 18928 & 0,00 & 0,00 & 0,00 & 4,257 & 0,851 \\
\hline 25 & 03 & 01/Nov & $11: 30$ & 18870 & 0,00 & 0,00 & 0,00 & 1,944 & 0,648 \\
\hline 26 & 05 & 06/Nov & $15: 00$ & 18776 & 0,697 & 0,00 & 0,697 & 4,202 & 0,840 \\
\hline
\end{tabular}

\section{Simulación}

Pareció interesante hacer una sencilla simulación de evaporaciones de suelo desnudo partiéndose de la Eto. Como la estación meteorológica más cercana está alejada $15 \mathrm{~km}$ del proyecto, su conjunto total de datos no es muy aplicable. Aparte, la modelación matemática no es aqui objetivo central. Por todo ello, se utilizarán solamente 3 métodos clasicos para estimar la evapotranspiración potencial que son: Tanque Clase A, Blaney-Criddle y Thornthwaite. Seria interesante aplicar um modelo más sofisticado como, por ejemplo, el de Ritchie (1972) para suelo desnudo pero, como no habia pluviógrafo cercano ni 


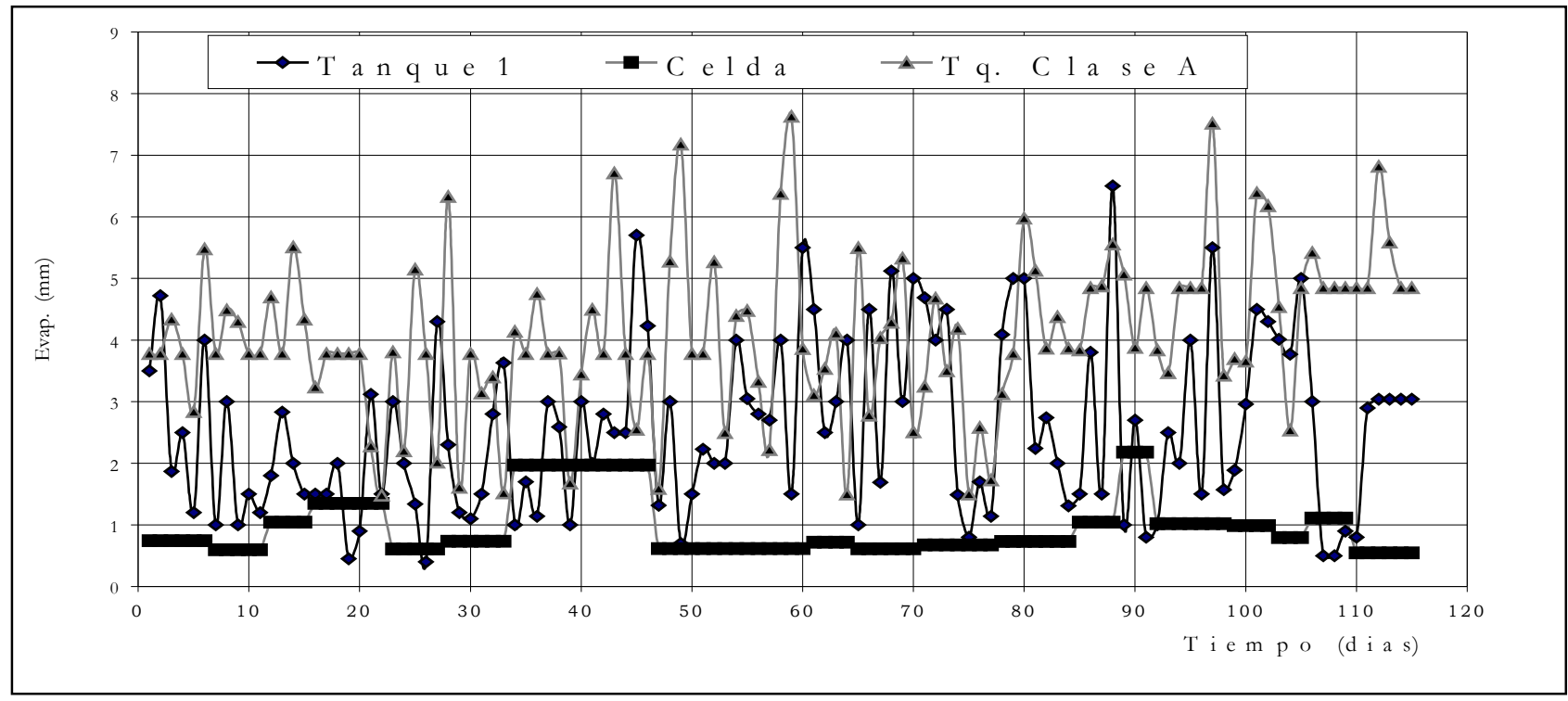

Figura 3 - Evaporaciones de la Celda, tanque 1 y tanque Clase A

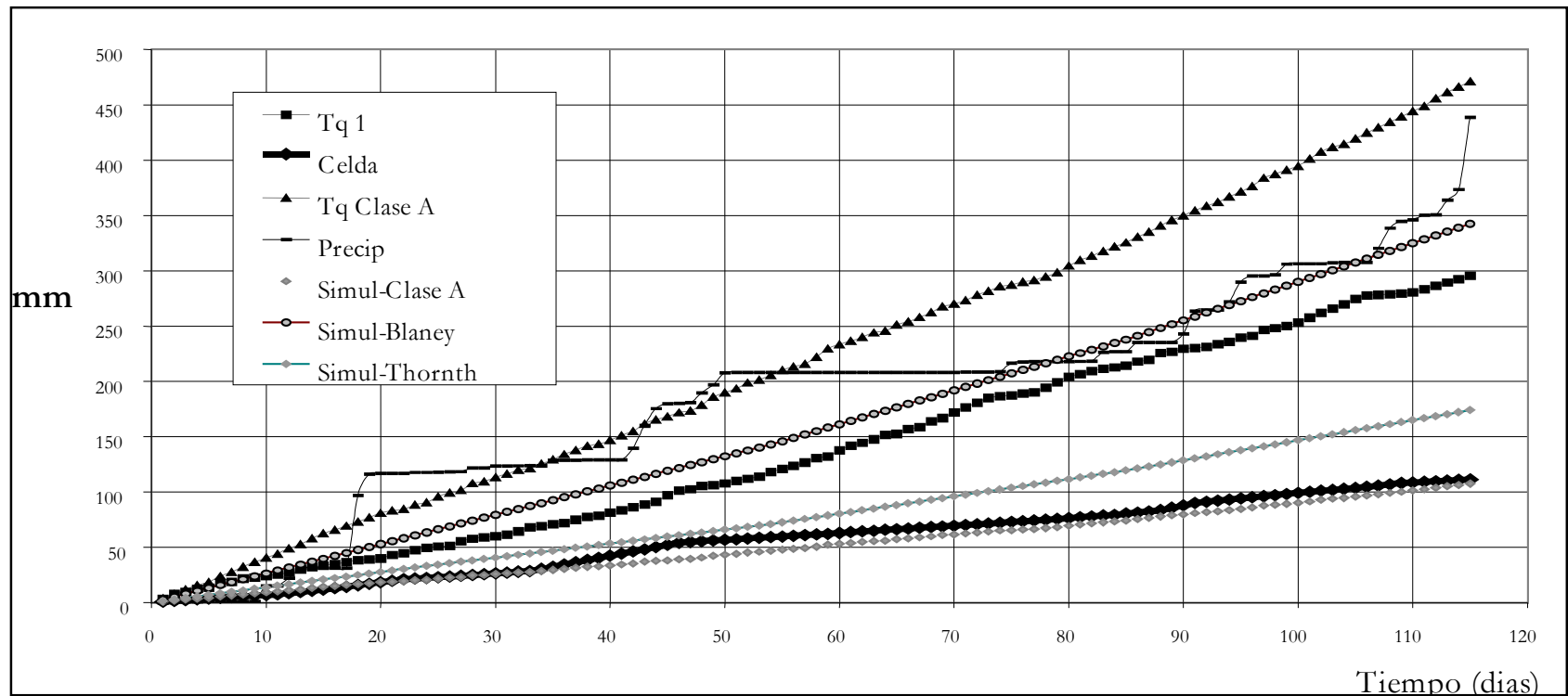

Figura 4 - Lluvia y Evaporaciones acumuladas

datos para identificar las "fases I y II" de la evaporación, se elegió tan solo solo aquellos 3 comentados. Para simular aproximadamente la evaporación real de suelo desnudo, se puede considerar que en los primeros dias después de una sembradura, la evapotranspiración que ocurre es praticamente la evaporación de dicho suelo desnudo (ver Figura 1). Con el método del tanque, se puede obtener la ETo y, después, la ETPc, (en este caso = ES) a través de..las ecuaciones (1) y (2). Doorenbos y Pruitt (1977) presentaron un método aproximado para determinar el coeficiente de tanque Clase A $(\mathrm{Kt})$ en función de las condiciones del suelo en las cercanias del tanque, velocidaddel viento, humedad relativa del aire y distancia del tanque hasta el final del césped o suelo desnudo. Para Florianópolis y al época del experimento, se tiene aproximadamente: H.R. $85 \%$; viento moderado entre 175 y $425 \mathrm{Km} \mathrm{día}^{-1}$; distancia hasta el borde entre 10 y 100 metros, con césped. Segundo el citado autor, "Kt" debería ser aproximada 
Tabla 3 - Comparación entre distintos autores

\begin{tabular}{|c|c|c|c|c|c|c|c|c|}
\hline \multirow[t]{2}{*}{$\mathbf{N}^{\mathbf{o}}$} & \multirow[t]{2}{*}{ Autor } & \multirow{2}{*}{$\begin{array}{l}\text { Precip } \\
\text { Anual } \\
(\mathrm{mm})\end{array}$} & \multirow{2}{*}{$\begin{array}{l}\text { Rango de } \\
\text { temp. } \\
\left({ }^{\circ} \mathrm{C}\right)\end{array}$} & \multirow[t]{2}{*}{ Suelo } & \multicolumn{3}{|c|}{$\begin{array}{l}\text { Evaporación diária } \\
\left(\mathrm{mm} \mathrm{dia}^{-1}\right)\end{array}$} & \multirow{2}{*}{$\begin{array}{l}\text { Vol. } \\
\text { del lisim. } \\
\text { ( litros) }\end{array}$} \\
\hline & & & & & Min & Média & Máx & \\
\hline 1 & $\begin{array}{l}\text { Este trabajo: Tanque } \\
\text { "Clase A" }\end{array}$ & 1683 & $08 / 28$ & Agua & 1,48 & 4,10 & 7,63 & 296,0 \\
\hline 2 & Este trabajo: Tanque 1 & 1683 & $08 / 28$ & Água & 0,40 & 2,57 & 6,50 & 9,0 \\
\hline 3 & Este trabajo: Celda & 1683 & $08 / 28$ & Arena gruesa & 0,55 & 0,97 & 2,18 & 21,0 \\
\hline 4 & $\begin{array}{l}\text { Este trabajo: Simulación } \\
\mathrm{C} / \mathrm{Kt}=0,76 \text { y } \mathrm{Kc}=0,30\end{array}$ & 1683 & $08 / 28$ & Arena gruesa & 0,34 & 0,93 & 1,74 & 296,0 \\
\hline 5 & $\begin{array}{l}\text { Mellouli et al (2000)*** } \\
\text { [Tunisia y España] }\end{array}$ & 190 & $20 / 30$ & Suelo arenoso & $\mathrm{n} / \mathrm{d}$ & 1,25 & $\mathrm{~N} / \mathrm{d}$ & 118,0 \\
\hline 6 & $\begin{array}{l}\text { Mellouli et al (2000)*** } \\
\text { [Tunisia y España] }\end{array}$ & 280 & $20 / 30$ & Suelo arenoso c/ rocas & $\mathrm{n} / \mathrm{d}$ & 0,79 & $\mathrm{n} / \mathrm{d}$ & 118,0 \\
\hline 7 & $\begin{array}{l}\text { Menziani et al (1999) } \\
\text { [talia] }\end{array}$ & 718 & $12 / 28$ & Arena fina & 1,77 & 2,74 & 4,60 & 3141,6 \\
\hline 8 & $\begin{array}{l}\text { Jackson et al (1999) } \\
\text { [Kenia] }\end{array}$ & 782 & $15 / 30$ & Suelo areno-arcilloso & 1,03 & 1,92 & 2,80 & 2,0 \\
\hline 9 & $\begin{array}{l}\text { Mwendera et al (1997) } \\
\text { [Belgica] }\end{array}$ & 720 & $05 / 24$ & Suelo limoso (lavrado) & 0,10 & 0,64 & 1,75 & 5,0 (micro) \\
\hline 10 & $\begin{array}{l}\text { Mwendera et al (1997) } \\
\text { [Belgica] }\end{array}$ & 720 & $05 / 24$ & $\begin{array}{l}\text { Suelo limoso (lavrado y } \\
\text { rejado) }\end{array}$ & 0,10 & 0,52 & 1,65 & 5,0 (micro) \\
\hline 11 & $\begin{array}{l}\text { Plauborg (1995) } \\
\text { [Dinamarca] }\end{array}$ & 600 & $0 / 15$ & Suelo arenoso & 1,30 & 1,65 & 2,00 & 0,8 (micro) \\
\hline 12 & $\begin{array}{l}\text { Wallace et al (1999) } \\
\text { [Kenia] }\end{array}$ & 782 & $15 / 35$ & $\begin{array}{l}\text { Suelo areno arcilloso } \\
\text { (bajo sombra de árbol) }\end{array}$ & 0,35 & 0,92 & 4,60 & 1,8 (micro) \\
\hline 13 & $\begin{array}{l}\text { Wallace et al (1999) } \\
\text { [Kenia] }\end{array}$ & 782 & $15 / 35$ & Suelo areno arcilloso & 0,45 & 1,33 & 6,10 & 1,8 (micro) \\
\hline 14 & $\begin{array}{l}\text { Qiu et al (1998)*** } \\
\text { [Japon] }\end{array}$ & 2074 & $08 / 40$ & Arena gruesa & 0,30 & 0,97 & 1,65 & 18,0 y 78,0 \\
\hline 15 & $\begin{array}{l}\text { Pauliukonis et al (2001) } \\
\text { [USA] }\end{array}$ & 1080 & $16 / 31$ & Suelo vegetal \# & 0,40 & 2,03 & 3,50 & 34,3 \\
\hline 16 & $\begin{array}{l}\begin{array}{l}\text { Yuen et al (2001)* } \\
\text { [Australia] }\end{array} \\
\end{array}$ & 874 & $09 / 23$ & Arcilla & 0,05 & 1,20 & 2,27 & $\begin{array}{l}\text { Relleno } \\
\text { real }\end{array}$ \\
\hline 17 & $\begin{array}{l}\text { Yuen et al (2001)** } \\
\text { [Australia] }\end{array}$ & 874 & $09 / 23$ & Arcilla & 0,05 & 0,98 & 2,40 & $\begin{array}{l}\text { Relleno } \\
\text { Real }\end{array}$ \\
\hline 18 & $\begin{array}{l}\text { Daamen et al (1995) } \\
\text { [Niger] }\end{array}$ & 540 & $20 / 40$ & Arena media & 0,43 & 1,71 & 3,55 & 1,8 (micro) \\
\hline
\end{tabular}

Las máx. y mín. refiérense, respect., a "Fase I" (saturado) y Fase II" (secando). / * calculado con modelo Jensen / ** calc. con modelo H.E.L.P./ *** c/ lluvia artificial / \# autor no indica su clasificación / "n/d" ato no disponible/ “micro” micro lisimetro

mente 0,76 como siendo lo más adecuado para el caso de la estación presente. Aqui, se hizo una simplificación considerándose ese coeficiente como constante durante todo el monitoreo. En general en el estadio inicial de una cultura, Kc varia de 0,25 a 0,65. Se adoptó aqui el valor constante $K c=0,30$.
Según Blaney-Criddle

$$
\text { Eto }=a+b[(p(0,46 t+8)]
$$

Para Thornthwaite:

$$
\mathrm{ETP}=16 \mathrm{f}_{\mathrm{c}}(10 \mathrm{t} / \mathrm{I})^{\mathrm{c}}
$$




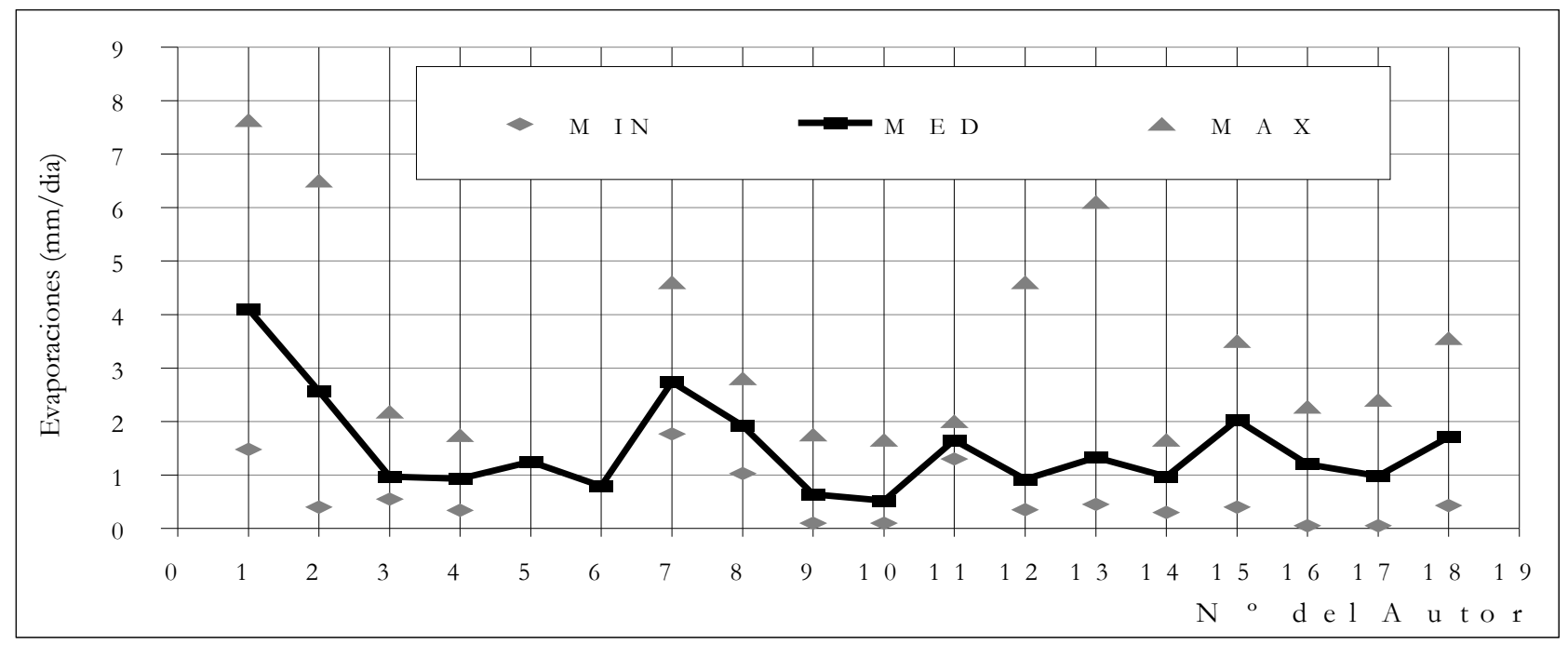

Figura 5 - Contraste con otros autores

$$
\begin{gathered}
\mathrm{Ic}=\sum_{1}^{12}\left(\mathrm{t}_{\mathrm{i}} / 5\right)^{1,514} \\
\mathrm{c}=67,5 \cdot 10^{-8} \mathrm{Ic}^{3}-7,71 \cdot 10^{-6} \mathrm{Ic}^{2}+0,01791 \mathrm{Ic}+0,492(7)
\end{gathered}
$$

En en primer método (tanque Clase A) el cálculo fue determinado dia a dia. En los dos últimos, en base mensual pero después considerando una distribución uniforme dentro de cada mes. Las evaporaciones del água del tanque 1 resultaron praticamente coincidentes con las mediciones de la celda. La simulación con evapotranspiración potencial de Blaney-Criddle resultó demasiadamente alta pero la de Thornthwaite más aproximada con las observaciones de la celda. La evaporación del tanque Clase A se mostró con una pendiente casi constante $\left(4,10 \mathrm{~mm} \mathrm{dia}^{-1}\right)$, sin depender mucho de las lluvias ocurridas. Ya el tanque 1 tube comportamiento similar, con menor pendiente y con alguna sensibilidad a más. Quizás por su reducido tamaño.

La Tabla 1 ya presentada incluye los principales resultados.

\section{Comparaciones con otros trabajos}

La Tabla 3 presenta un resumen general de resultados de varios autores y La Figura 5, valores máximos médios y mínimos. En abcisas, se ubicó los distintos trabajos identificados por el número de ordem. De todo este artículo, Yuen et al (2001) presentó el mínimo valor con 0,05 $\mathrm{mm} \mathrm{dia}^{-1}$ y Wallace et al (1999), el máximo, con 6,10 $\mathrm{mm} \mathrm{dia}{ }^{-1}$. Hay que notar que el primero autor utilizó arci- lla y ese último, arena arcillosa. Es decir, cuanto mayor el diámetro, más fácil es la evaporación. Se nota que hay una gran variación en los volúmenes de los lisimetros utilizados. Incluso, se pone de manifiesto la utilización de los "micro-lisímetros" con volúmenes hasta 0,80 litros. La celda del presente artículo tube un volumen de 21 litros. La misma Tabla 3 enseña lluvias anuales pero su influencia no pudo ser verificada pues los períodos monitoreados por cada autor varian entre las estaciones. Los rangos de temperatura del aire si muestran tendencia a influir en las evaporaciones tanto médias como máximas. Por ejemplo, el ya citado Wallace et al (1999) en Kenia presentó la máxima evaporación con temperaturas llegando hasta un $35^{\circ}$ C. En Dinamarca Plauborg (1995), con temperaturas de 0 a $15^{\circ} \mathrm{C}$, presentó media de 1,65 y máxima de $2,00 \mathrm{~mm}$ día ${ }^{-1}$. Es decir, resultados algo regulares. Por otro lado, como se puede ver, los otros resultados no aclaran esa tendencia. Mellouli et al (2000), Qyu et al (1998) y Yuen et al (2001) contrastaron datos reales con los modelos matemáticos Jensen et al $(1071.1979,1990)$ y HELP (Schroeder et al 1994).

\section{CONCLUSIONES}

Se presentó aqui una utilización de un pequeño lisímetro portable de pesaje para la medición de evaporación real de suelo desnudo no saturado de la cobertura arenosa de un modelo reducido le relleno sanitário. La camada de basura abajo del suelo no pareció influir de manera significativa en los resultados que se presentan bien ubicados dentro del rango de resultados de otros trabajos sin la dicha basura. La balanza utilizada tube la 
capacidad máxima de $50 \mathrm{~kg}$ y sensibilidad de 2,0 gramos. Como el peso de la celda varió aproximadamente en el rango de 18 a $21 \mathrm{Kg}$, la precisión média fue de 0,01\% considerada excelente.

\section{RECOMENDACIONES}

Considerando la carga máxima de la balanza utilizada y su excelente precisión, se puede aumentar el tamaño de la celda y disminuir un poco los períodos de tiempo entre dos mediciones consecutivas. Asi, los resultados tendrán un ajuste fino casi a diario y serán más representativos de la realidad de los rellenos sanitários.

\section{AGRADECIMIENTOS}

Agradezco al PROSAB III - Programa de Saneamento Básico y al Laboratório de Hidráulica del Departamento de Ingeniería Sanitária y Ambiental de la UFSC donde se realizó el diseño, utilizó materiales y se construyó el aparato. Por último, al prof. Marciano Maccarini del Depto de Ingeniería Civil de la UFSC por la utilización de una excelente balanza electrónica de precisión .

\section{REFERENCIAS}

BLANEY, H. F.; CRIDDLE,W.D. (1950) Determining Water Needs From Climatological Data. USDA, SCS / SOSPT, pp8-9.

CAPELO NETO, J.; MOTA, S.; SILVA, F.J.A. da (1999). Geração de Percolado em Aterro Sanitário no Semi-árido Nordestino: uma Abordagem Quantitativa, R. E. S.e Ambiental, V. 4, n 3, 160-167, Rio de Janeiro.

DAAMEN, C.C.; SIMMONDS, L.P.; SIVAKUMAR, M.V.K. (1995) The impact of sparse millet crops on evaporation from soil in semi-arid Niger. Agricultural Water Management, 27(1995)225-242.

DOORENBOS, J. ; PRUITT (1977). Guidelines for Predicting Crop Water Requirements.FAO Irrig. Drain. Papern ${ }^{\circ} 24$, p 1-179.

FENN, D.G., HANLEY,K.J., DeGEORE, T. V.(1975). Use of Water Balance Method for Predicting Leachate Generation for Solid Waste Disposition Sites, EPA Report $n^{\circ} \mathrm{EPA} / 530 / \mathrm{SW} 168$, Cincinnati.

FUNGAROLI, A . A ., STEINER, R.L.(1971); Laboratory Study of the Behavior of a Sanitary Landfill, Journal of the WPCF, v. 43, no 2, Feb., p 252-267.

GEE, J.R.(1981), Prediction of Leachate Accumulation in Sanitary Landfills, Applied Research \& Practice on Municipal and Industrial Waste , 4 Conf. Madison.

HARGREAVES, G. H..(1994) Defining and Using Reference Evapotranspiration, Journal of Irrigation and Drainage Engineering, vol. 120, p.1132-9.
JACKSON, N. A .; WALLACE, J.S. (1999), Soil Evaporation Measurementas in an Agroforestry System in Kenia, Agricultural and Forest Meteorology 94 (1999)203-215.

JENSEN, M.E; ROBB, D.C.N.; FRANZOI, C. E.(1970), Scheduling Irrigation Using Climate-Crop-Soil Data, J. Irrig. Drain. Div. Proc.ASCE- 96 (IR2), 25-38.

JENSEN, M.E; WRIGHT; J.L.; PRATT, B. L. (1971), Estimating Soil Moisture Depletion from Climate, Crop and Soil Data. Tr.Am. S.Agr.Eng 14(5), 954-959.

JENSEN, M.E; BURMAN, R. D.; ALLEN, R.G. (1990), Evapotranspiration and Irrigation Water Requirements, ASCE Manuals and Reports on Engineeering Practice no. 70, ASCE.

JENSEN, M.E.; HAISE, H.R. (1963). Estimation of Evapotranspiration from Solar Radiation.J. Irrig. Drain. Div., v89, p.15-41.

JENSEN, M.E.(1980) Design and Operation of Farm Irrigation Systems. A .S.A .E., St. Joseph.

MAURER, R. W. (1994), A paradigm shift from storage to bioreactors. Proc.Conf. "Landfill Tomorrow", Center of ECWM, I. College, London, pp. 1-5.

MEDEIROS, P. A ; CASTILHOS Jr. A . B de; OLIVEIRA, J.C.S.; SOARES, S.R (2002),Geração de Líquidos Percolados em Resíduos com Cobertura Permeável,VIS..Ítalo/Bras.deEng.S.e Amb.,Vitória.

MEDEIROS, P. A . ; SILVA, J. D. da; CASTILHOS Jr, A . B. de (2002), Balanço Hídrico em Aterros de Resíduos Sólidos Urbanos - Escala Experimental de Laboratório, in: Alternativas de Disposição de Resíduos Sólidos Urbanos para Pequenas Comunidades- Coletânea de Trabalbos Técnicos, Castilhos Jr. A . B. de; Lange, L.C.; Gomes, L. P.; Pessin, $\mathrm{N}$. (organizadores), PROSAB 3, Florianópolis.

MEDEIROS, P. A .; CASTILHOS Jr, A . B. de .; SILVA, J.D.da; DELAZARI, P.V. A. (2002), Monitoreo del Balance Hídrico en Basura Domiciliaria Urbana, III Simpósio Internacional de Qualidade Ambiental / Gestão Ambiental Urbana e Industrial, Pôrto Alegre.

MELLOULI, H.J.; WESEMAEL B. V.; POESEN, J.; HARTMANN, R. (2000); Evaporation Losses From Bare Soil as Influenced by Cultivation Techniques in Semi-Arid Regions. Agricultural Water Management, 42(2000)355369.

MENZIANI, M.; PUGNAGHI S. ; PILAN, L. SANTANGELO, R.; VINCENZI S.(1999) .Field Experimentation to Study Evaporation from a Saturated Bare Soil. Phys. Chem. Earth (B), vol. 24, no.7,pp 813-818.

MWENDERA, E.J. FEYEN, J.(1997). Tillage and Evaporativity Effects on the Drying Characteristics of a Silty Loam: Evaporation Prediction Models.Soil and Tillage Research 41 (1997) 127-140.

NERILO, N.; MEDEIROS, P. A ., CORDERO, A . (2002), Chuvas Intensas no Estado de Santa Catarina, Editora da UFSC / EdiFURB, 156 p. Florianópolis, 
PAULIUKONIS, N.; SCHNEIDER, R. (2001) Temporal Patterns in Evapotranspiration from lysimeters with three Commom Wetland Plant Species in the Eastern USA, Aquatic Botany, 71(2001)35-46.

PENMAN, H.L.(1948). Natural Evaporation From Open Water,Soil and Grass. Proc. Royal Society. Ser. A, n. 193

PLAUBORG, F. (1995); Evaporation From Bare Soil in a Temperate Humid Climate-Measurement Using MicroLysimeters and Time Domain Reflectometry, Agricultural and Forest Meteorology 76 (1995)1-17.

QIU, G. Y.; YANO, T.; MOMII, K. (1998).An Improved Methodology to Measure Evaporation from Bare Soil Based on Comparison of Surface Temperature with a Dry Soil Surface.Journal of Hydrology.210(1998) 93-105.

RICHIE, J. T. (1972) Model for predicting evaporation from a row crop with incomplete cover. W.Res. 8.

SCHROEDER, P. R.; DOZIER, T.S.; ZAPPI, P.A .: Mc ENROE, B. M. ; SJOSTROM, J. W. , PEYTON R.L.(1994), The (HELP) Model: Eng Doc.for Vers 3 (EPA/600/R-94/168b)PA/ORD, Washington, DC.

THORNTHWAITE, C. W.; HOLZMAN, B. (1939) The Determination of Evaporation from Land and Water Surfaces. Montlhy Weather Review, v.67 p $4-11$.

TUCCI, C. E. M.( 1993), (org.), Hidrologia- Ciência e Aplicação, , UFRGS, EDUSP, ABRH, Pôrto Alegre.

WALLACE, J.S.; JACKSON, N. A.; (1999), ONG, C.K. Modelling Soil Evaporation in na Agroforestry System in Kenia., Agricultural and Forest Meteorology 94 (1999)184202.

\title{
Cell for Monitoring Actual Unsatured Bare Soil Evaporation.
}

\begin{abstract}
This paper deals with unsatured bare soil evaporation and complements previous laboratory research on water balance of domestic solid waste sanitary landfills. A portable small cell was designed and developed specifically to measure that evaporation through successive weightings utilizing a digital scale while water evaporation was monitored through a small evaporimeter tank. The results obtained from both devices were compared to nearest Class $A$ tank historical data series. Evaporation was also calculated through three simple mathematical simulations using suitable tank and first stage crop coefficients. A literature comprehensive search enabled a general comparision of the results under many climates. Despite its low cost, the portable cell delivered satisfactory performance.
\end{abstract}

Key-Words: bare soil evaporation; physical models; lysimeters. 